\title{
Use of Adrenomedullin Derivatives for Molecular Imaging of Pulmonary Circulation
}

François Harel ${ }^{1,2}$, Yan $\mathrm{Fu}^{3}$, Quang Trinh Nguyen ${ }^{1}$, Myriam Letourneau ${ }^{3}$, Louis P. Perrault ${ }^{1,4}$, Alexandre Caron ${ }^{1}$, Alain Fournier ${ }^{3}$, and Jocelyn Dupuis ${ }^{1,4}$

${ }^{I}$ Research Center, Montreal Heart Institute/Université de Montréal, Montreal, Quebec, Canada; ${ }^{2}$ Department of Radiology, Université de Montréal, Montreal, Quebec, Canada; ${ }^{3}$ Institut National de la Recherche Scientifique-Institut Armand Frappier, Pointe-Claire, Quebec, Canada; and ${ }^{4}$ Department of Medicine, Université de Montréal, Montreal, Quebec, Canada

Currently, there is no low-molecular-weight agent for imaging of the pulmonary circulation. Adrenomedullin (AM) is a peptide predominantly cleared by the pulmonary circulation through specific endothelial receptors. We developed human AM derivatives radiolabeled with ${ }^{99 m T c}$ and evaluated their biodistribution, plasma kinetics, and utility as pulmonary vascular imaging agents. Methods: Two derivatives radiolabeled with $99 \mathrm{mTc}$ were evaluated: the natural cyclic form of the peptide, to which the chelator diethylenetriaminepentaacetic acid was added (C-DTPA-AM), and the linear form, which allows direct labeling (L-AM). The compounds were injected into dogs, and the activities of the tracers in blood and in organs were determined with a nuclear medicine camera. Single-pass pulmonary clearance was measured by the indicator dilution technique. The capacity to image perfusion defects was evaluated after surgical pulmonary artery ligation. Results: Both derivatives were rapidly cleared from plasma, with elimination half-lives of 42 and 32 min for C-DTPA-AM and L-AM, respectively. The lungs retained most of the activity after $30 \mathrm{~min}$; this activity was higher $(P=0.02)$ for L-AM $(42 \% \pm 5 \%$ [mean \pm SEM]) than for C-DTPA-AM $(27 \% \pm 1 \%)$. Lung activity slowly declined over time but was maintained after $2 \mathrm{~h}$ at approximately $20 \%$ for both tracers. The single-pass pulmonary clearance of plasma L-AM was $414 \pm 85 \mathrm{~mL} / \mathrm{min}$. There was a higher level of urinary excretion of L-AM than of C-DTPA-AM. After pulmonary artery ligation, perfusion defects were easily detectable by external imaging. Conclusion: AM derivatives are promising compounds for molecular imaging of the pulmonary circulation. L-AM displayed higher levels of initial lung retention and of kidney excretion.

Key Words: radiopharmaceutical; peptide; receptor; biodistribution; lung

J Nucl Med 2008; 49:1869-1874

DOI: 10.2967/jnumed.108.054023

H uman adrenomedullin (AM) is a 52-amino-acid multifunctional regulatory peptide that is widely distributed in various tissues and highly expressed in endothelial cells

\footnotetext{
Received May 1, 2008; revision accepted Aug. 7, 2008.

For correspondence or reprints contact: Jocelyn Dupuis, Research Center, Montreal Heart Institute, 5000 Bélanger St., Montreal, Quebec, Canada H1T $1 \mathrm{C8}$.

E-mail: jocelyn.dupuis@bellnet.ca

COPYRIGHT @ 2008 by the Society of Nuclear Medicine, Inc.
}

(1-3). Plasma levels of AM are increased in various acute and chronic cardiovascular disorders, such as myocardial infarction and heart failure, as well as in many other pathologic conditions, such as pulmonary hypertension and parenchymal lung diseases $(4,5)$. The predominant biologic effect of AM is vasodilatation, including the pulmonary circulation (6), and this endogenous peptide is currently being considered for its beneficial therapeutic potential in cardiovascular disorders $(4,5)$.

Recent studies have established that circulating AM is predominantly cleared by the pulmonary circulation through specific endothelial receptors (7-9). The AM receptor is a heterodimer composed of the calcitonin receptor-like receptor and receptor activity-modifying protein 2 , both of which are highly expressed in the lungs $(1,5)$. The AM receptor is abundantly expressed in human alveolar capillaries and in rat pulmonary microvascular endothelial cells $(10,11)$. Thus, human and rat lungs contain specific AM-binding sites $(12,13)$ at a density higher than that in any other organ studied (13).

On the basis of this evidence, we hypothesized that AM derivatives labeled with an externally detectable agent could be used to image the pulmonary circulation. Hence, we developed a chelated derivative of the natural cyclic form of human AM by using diethylenetriaminepentaacetic acid (DTPA) as a chelating agent (C-DTPA-AM), allowing radiolabeling with ${ }^{99 \mathrm{~m}} \mathrm{Tc}$. We also evaluated the linear form of the peptide (L-AM), which allows direct labeling without the need for a chelator. The present study was designed to systematically evaluate the biodistribution, pharmacokinetics, and multiorgan clearance of these two ${ }^{99 \mathrm{~m} T \mathrm{Tc}-\text { labeled AM }}$ derivatives in dogs in vivo. Furthermore, the capacity of these molecular imaging agents to diagnose perfusion defects in the pulmonary circulation was evaluated after surgical pulmonary artery ligation.

\section{MATERIALS AND METHODS}

\section{Synthesis and Purification of AM Derivatives}

Human AM is a cyclic 52-amino-acid peptide comprising 2 cysteine residues located at positions 16 and 21 from the $\mathrm{N}$ terminus. The 2 cysteine residues are bound by a disulfide linkage, forming a loop composed of 6 amino acids. The linear derivative 
used in the present study (L-AM) has the same structure, except for the lack of a loop because of the omission of the disulfide linkage. This open linear form allows direct labeling with ${ }^{99 \mathrm{~m}} \mathrm{Tc}$ at the level of the available cysteine residues. The cyclic derivative used here (C-DTPA-AM) is identical to native human AM, except for the addition of DTPA as a ${ }^{99 \mathrm{~m}} \mathrm{Tc}$ chelator at the $\mathrm{N}$ terminus.

L-AM was synthesized by use of a solid-phase procedure based on fluorenylmethyloxycarbonyl chemistry with a Rink-AM-amide resin (Chem-Impex International) as the solid support.

Disulfide bond formation to produce C-DTPA-AM was performed by overnight air oxidation after dissolution of the linear peptide in an aqueous solution $(\mathrm{pH} 8.8)$ at a concentration of $0.1 \mathrm{mg} / \mathrm{mL}$. The chelating moiety was incorporated in the peptide chain using DTPA dianhydride (Sigma-Aldrich) after a deprotection step of the amino terminus. Peptides were purified by reversed-phase high-performance liquid chromatography (RPHPLC) with a flanged MODCOL column $(25 \times 3.5 \mathrm{~cm})$ packed with a Jupiter $\mathrm{C}_{18}$ matrix ( $15 \mu \mathrm{m}, 30 \mathrm{~nm}$ ) (Phenomenex).

Purity was evaluated by analytic RP-HPLC, and mass was established by matrix-assisted laser desorption ionization-time-offlight mass spectrometry (Voyager DE; Applied Biosystems).

\section{Radiolabeling and Purification}

Sample vials containing a $2.9 \mathrm{nM}$ concentration of either C-DTPA-AM or L-AM were kept at $-20^{\circ} \mathrm{C}$. Radiolabeling was performed by adding to the vial $100 \mu \mathrm{L}$ of $\mathrm{HCl}(1 \mathrm{mM})$ and $14.8 \mu \mathrm{L}$ of $\mathrm{SnCl}_{2} \cdot 2 \mathrm{H}_{2} \mathrm{O}(0.2 \mathrm{mg} / \mathrm{mL}, 13 \mathrm{nM})$. Immediately after dissolution of the materials, $555 \mathrm{MBq}$ of freshly prepared $\mathrm{Na}^{99 \mathrm{~m}} \mathrm{TcO}_{4}(28.9 \mathrm{pM})$ in saline solution was added, and the mixture was kept at room temperature for $1 \mathrm{~h}$. After the radiolabeling step, $1 \mathrm{~mL}$ of phosphate-buffered saline $(\mathrm{pH}$ 7.4) was added to buffer the solution.

The ${ }^{99 m}$ Tc-C-DTPA-AM solution was purified with an RPHPLC system (Millipore/Waters). The radiochemical purity, as evaluated by instant thin-layer chromatography, was $93 \% \pm 2 \%$ (mean \pm SEM).

The ${ }^{99 m}$ Tc-L-AM solution was purified with a Sep-Pak cartridge (Waters). The radiochemical purity, as measured by instant thin-layer chromatography with ITLC-SG strips from PALL Life Sciences (PALL Corp.), was $\geq 95 \%$.

The average activity injected in the experiments was $148 \pm 41$ $\mathrm{MBq}$, and the specific activity was $52 \pm 15 \mathrm{MBq} / \mathrm{nmol}$ of derivative.

\section{Anesthesia and Animal Preparation}

All experimental procedures were performed in accordance with the regulations and ethical guidelines of the Canadian Council for the Care of Laboratory Animals and were approved by the Animal Ethics and Research Committee of the Montreal Heart Institute.

\section{Plasma Kinetics and Biodistribution of C-DTPA-AM and L-AM}

Mongrel dogs weighing 20-30 kg and testing negative for Dirofilaria immitis were anesthetized with intravenous sodium pentobarbital $(50 \mathrm{mg} / \mathrm{kg})$, intubated, and mechanically ventilated. Cutaneous electrocardiographic leads were installed, and 18-French intravenous catheters with 3-way stopcocks were inserted into both saphenous veins for injection and blood collection. Another catheter was inserted into the right femoral artery for continuous blood pressure monitoring. The animals were studied after intravenous injection of $18.5 \mu \mathrm{g}(\approx 185 \mathrm{MBq})$ of the labeled peptides ( $n=6$ for C-DTPA-AM and $n=7$ for L-AM).

\section{Pulmonary Artery Ligation}

Pulmonary perfusion defects were created by surgical ligation of pulmonary arteries. Four animals were prepared as described earlier, and anesthesia was maintained with $1 \%-3 \%$ isoflurane. After a medial thoracotomy, a lobar pulmonary artery was identified and ligated.

\section{Single-Pass Pulmonary Clearance of L-AM}

For precise quantification of the single-pass (first-pass) pulmonary clearance of L-AM, 8 dogs were prepared as described earlier. Transpulmonary indicator dilution curves were obtained with Evans blue dye-labeled albumin as a plasma reference as previously described in detail (14).

\section{In Vivo Biodistribution of C-DTPA-AM and Multiorgan Clearance}

Biodistribution was evaluated with a mobile Anger camera (Technicare) or with a dual-head e-cam (Siemens Medical Systems). A low-energy, high-resolution collimator was used for acquisition with a $20 \%$ energy window centered on $140 \mathrm{keV}$. A dynamic acquisition including regions of the lungs, heart, liver, and kidneys was recorded for $30 \mathrm{~min}$ (one frame per second during the first minute and then one frame per minute for the remaining time) with $256 \times 256$ matrices at $2.4 \mathrm{~mm}$ per pixel. Static acquisitions were also recorded for whole individual organs (lungs, kidneys, liver, heart, bladder, and gallbladder) at 30, 60, and $120 \mathrm{~min}$ after the initial injection with $256 \times 1,024$ matrices.

Dynamic and static acquisitions were evaluated with Matlab version 7.01 image analysis tool software (The MathWorks, Inc.). The ${ }^{99 \mathrm{~m}} \mathrm{Tc}$ total count, mean count, and region of interest comprising the whole organ were calculated for each organ. Data correction was applied for radioactive decay, table correction (dorsal images only), geometric mean, and organ attenuation based on the transmission factor. Results were expressed as a percentage of the total radioactivity injected.

\section{Binding Studies with Human Cells}

The binding of L-AM and C-AM was evaluated with a human breast adenocarcinoma cell line (MCF-7). These cells express approximately 50,000 AM receptors per cell (15). For each peptide, competitive-binding experiments were performed in triplicate with ${ }^{125}$ I-AM (amino acids $1-52$ ).

\section{Statistical Analysis}

Data for plasma kinetics were fitted by use of a 2-phase exponential-decay equation with GraphPad Prism version 4.0 software (GraphPad Software, Inc.). Differences between C-DTPA-AM and L-AM were evaluated with 2-tailed $t$ tests. A $P$ value of less than 0.05 was considered significant. All results were reported as mean \pm SEM.

\section{RESULTS}

\section{Plasma Kinetics of AM Derivatives}

Plasma levels of intravenously administered C-DTPAAM $(n=6)$ and L-AM $(n=7)$ rapidly decreased in accordance with a 2-compartment model, with a rapid distribution half-life of less than 2 min and elimination half-lives of $42 \mathrm{~min}$ (95\% confidence interval [CI], 30-68) and $32 \mathrm{~min}$ (CI, 22-61), respectively (Fig. 1). Compartmental analysis revealed that the ratios of rate constants for exchange between the central and the peripheral compart- 


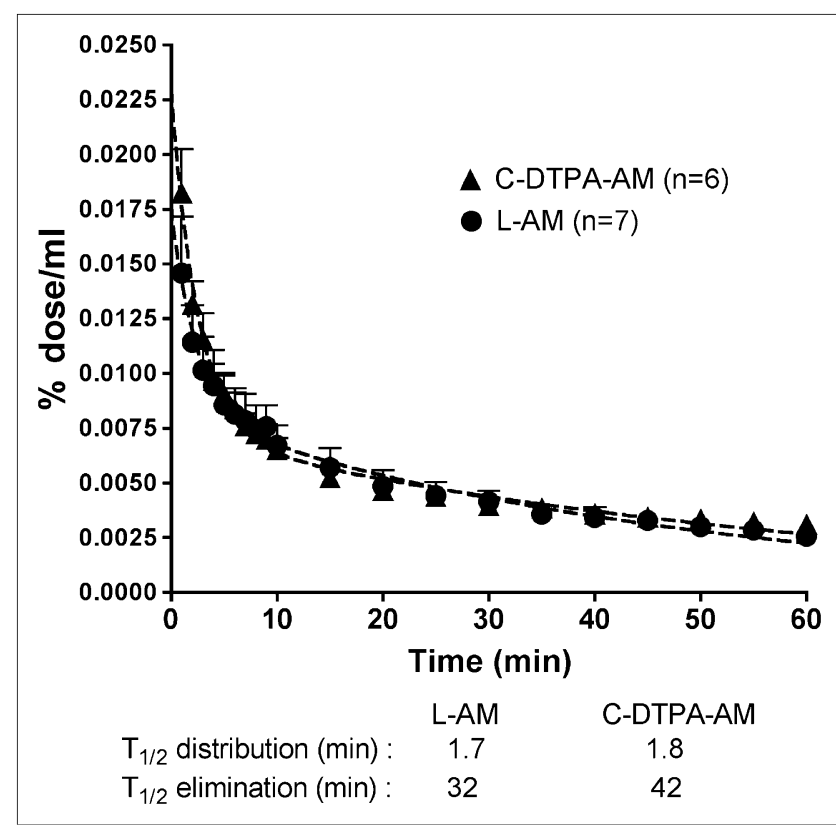

FIGURE 1. Plasma kinetics of ${ }^{99 m}$ Tc-labeled C-DTPA-AM and L-AM after single intravenous injections in dogs. Curve was fitted with 2-phase exponential decay equation. $\mathrm{T}_{1 / 2}=$ half-life.

ments were high, at 24 for C-DTPA-AM and 19 for L-AM, demonstrating an important distribution of drugs into the peripheral compartment.

\section{Biodistribution of AM Derivatives After Injection}

The biodistributions of the AM derivatives after 30 and $120 \mathrm{~min}$ are shown in Figures $2 \mathrm{~A}$ and $2 \mathrm{~B}$, respectively. After $30 \mathrm{~min}$, predominantly the lungs retained the peptides, with $27 \% \pm 1 \%$ of the injected dose for C-DTPA$\mathrm{AM}(n=6)$ and $42 \% \pm 5 \%$ for L-AM $(n=7)$. The lungs were followed in importance by the kidneys $(19 \% \pm 1 \%$ for C-DTPA-AM and $23 \% \pm 3 \%$ for L-AM) and the liver $(12 \% \pm 1 \%$ for C-DTPA-AM and $6 \% \pm 1 \%$ for L-AM). There was minimal uptake in other organs. Compared with the uptake of C-DTPA-AM, the uptake of L-AM was significantly higher in the lungs but lower in the liver and the heart.

Lung retention slowly declined over time but was maintained after $120 \mathrm{~min}$ at approximately $20 \%$ of the injected dose for both AM derivatives. The derivatives were excreted mainly by the kidneys, as evidenced by increasing kidney and bladder activities after $120 \mathrm{~min}$. The level of urinary excretion of L-AM was higher but the level of liver uptake of L-AM was lower after $120 \mathrm{~min}$.

An example of time-activity curves for the lungs, kidneys, liver, and heart of one animal after the injection of C-DTPA-AM is shown on a logarithmic time scale in Fig. 3. The labeled AM derivative was rapidly extracted by the lungs within the first minute after injection, with evidence of ongoing recirculatory uptake and a slow progressive decline in activity over $2 \mathrm{~h}$. The activity in the liver rapidly reached a plateau after only $3 \mathrm{~min}$. In the kidneys,

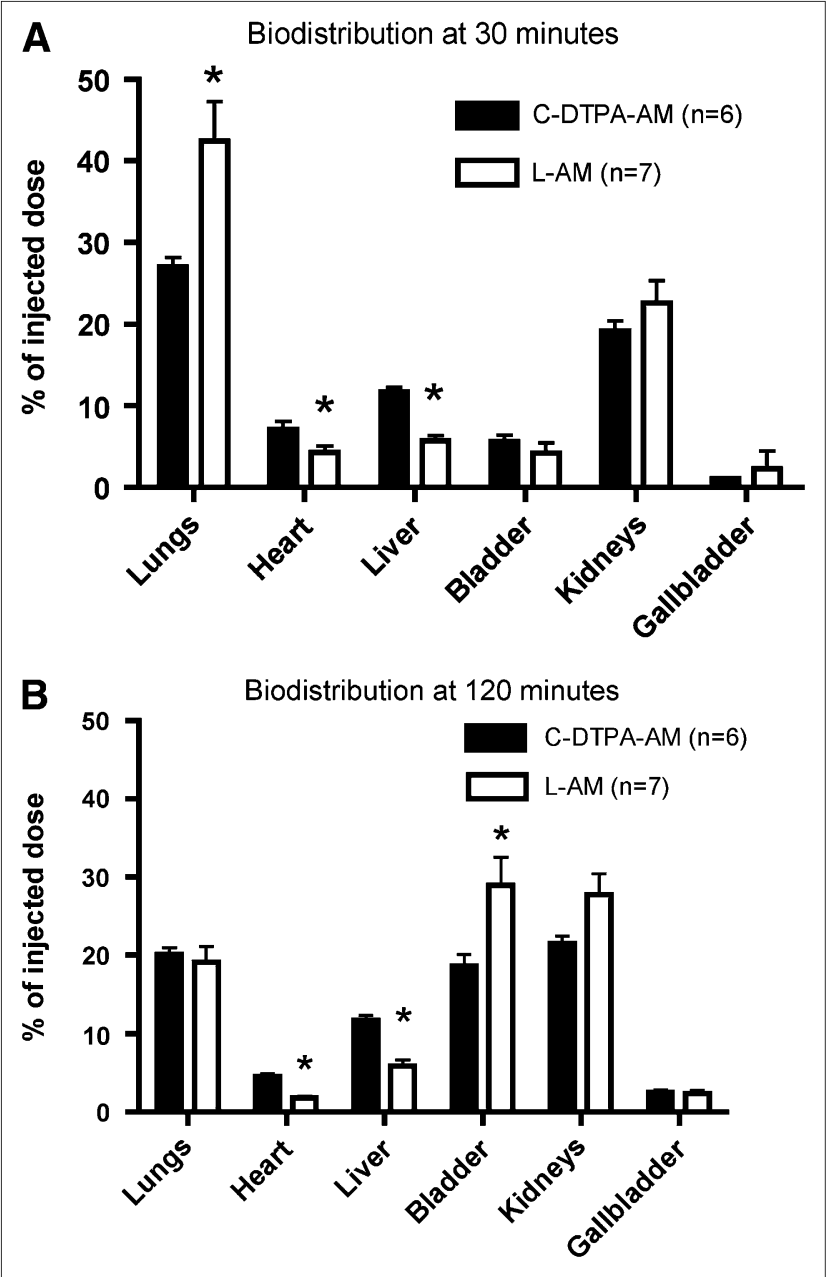

FIGURE 2. Organ biodistributions of ${ }^{99 \mathrm{~m} T c-l a b e l e d ~ C-D T P A-}$ $A M$ and L-AM $30(A)$ and 120 min (B) after intravenous injections in dogs. ${ }^{\star} P<0.05$ for L-AM vs. C-DTPA-AM.

there was a constant and progressive increase in activity over the 30 min of observation.

\section{Single-Pass Pulmonary Clearance of L-AM}

The capacity of the lungs to clear circulating AM from the circulation in vivo was determined with the indicator dilution technique in 8 animals; an example of an indicator dilution curve is shown in Figure 4. The single-pass pulmonary extraction of L-AM was $30 \% \pm 5 \%$, and the clearance of plasma L-AM by the pulmonary circulation was $414 \pm 85 \mathrm{~mL} / \mathrm{min}$.

\section{Imaging of Pulmonary Circulation with AM Derivatives}

An example of lung scintigraphic imaging after the injection of C-DTPA-AM is shown in Figure 5. Shortly after injection, the radiolabeled derivative was seen in the inferior vena cava and the right heart chambers. After $20 \mathrm{~s}$, there was mild activity in the heart region, and uptake by the lungs was already evident. After only $5 \mathrm{~min}$, stable, homogeneous, and good-quality imaging of the lungs was obtained. Lung activity persisted for up to $2 \mathrm{~h}$, and during 


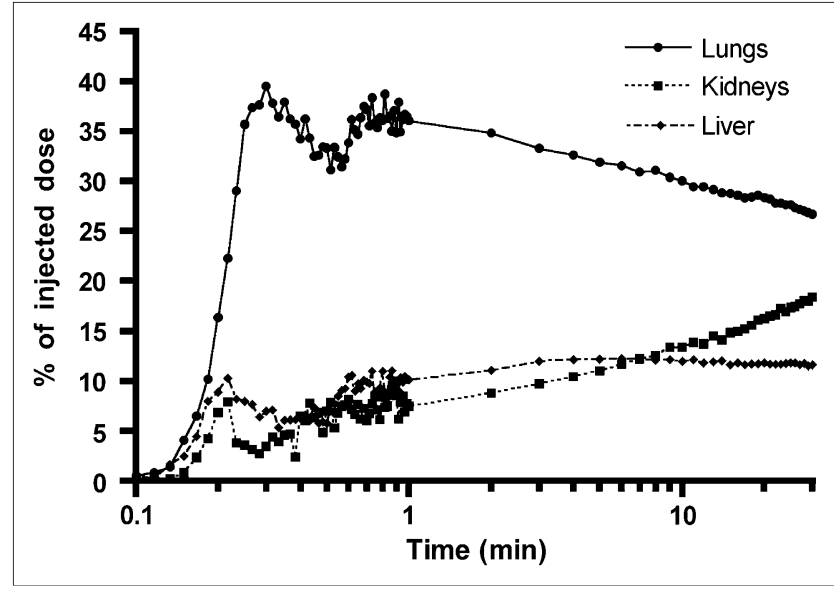

FIGURE 3. Organ kinetics of 99mTc-C-DTPA-AM after intravenous injection in a dog. Regions of interest for lungs, kidneys, and liver were continuously imaged for $30 \mathrm{~min}$. Time scale is logarithmic.

this time, the elimination of some tracer in the gallbladder became evident. The rapid and prolonged uptake of AM by the lungs represented an important advantage for the clinical development of this tracer. A whole-body scan obtained $30 \mathrm{~min}$ after the injection of L-AM into a dog is shown in Figure 6. Kidney elimination of the tracer was already evident at $30 \mathrm{~min}$ with some bladder activity.

To demonstrate that these new tracers could be used to image lung perfusion defects, we performed additional experiments after surgical ligation of lobar pulmonary arteries $(n=4)$. Corresponding anatomic perfusion defects were easily detectable after the injection of AM derivatives. An example of tomographic imaging of a perfusion defect with L-AM after ligation of the right middle lobar pulmonary artery is shown in Figure 7; tomographic imaging in a

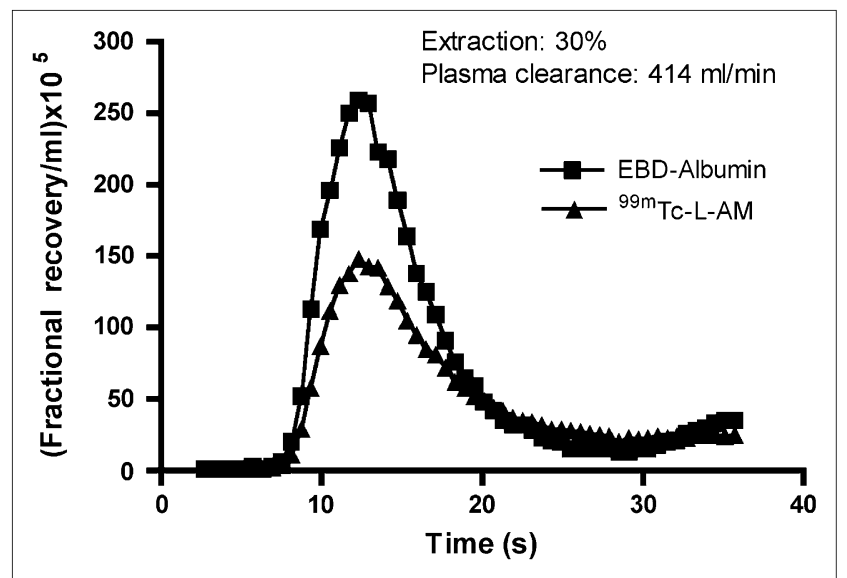

FIGURE 4. Example of indicator dilution experiment done to measure single-pass pulmonary clearance of L-AM. Concentrationtime curves for ${ }^{99 \mathrm{~m} T c-L-A M}$ and Evans blue dye-labeled albumin (EBD-Albumin) are shown. Differential area under curves represents mean single-pass extraction of L-AM by lungs.

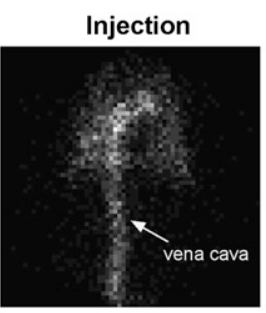

30 minutes

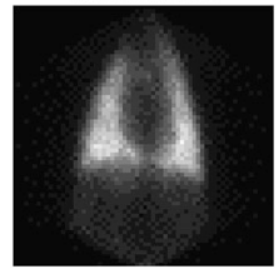

FIGURE 5. Lung perfusion scan with 99mTc-C-DTPA-AM. AM derivative was rapidly taken up by lungs, enabling imaging from 5 to $120 \mathrm{~min}$. Some gallbladder elimination was seen after $60 \mathrm{~min}$

control animal is shown for comparison. A typical wedgeshaped vascular defect was easily detectable.

\section{Competitive Binding to Human Cells}

Both C-DTPA-AM and L-AM displayed competitive binding to MCF-7 cells, with 50\% inhibitory concentrations

FIGURE 6. Whole-body scan of a dog 30 min after injection of $99 \mathrm{~m}$ Tc-L-AM. Tracer demonstrated homogeneous lung uptake, enabling good-quality imaging. There was evidence of kidney elimination with bladder activity as well as some liver activity.






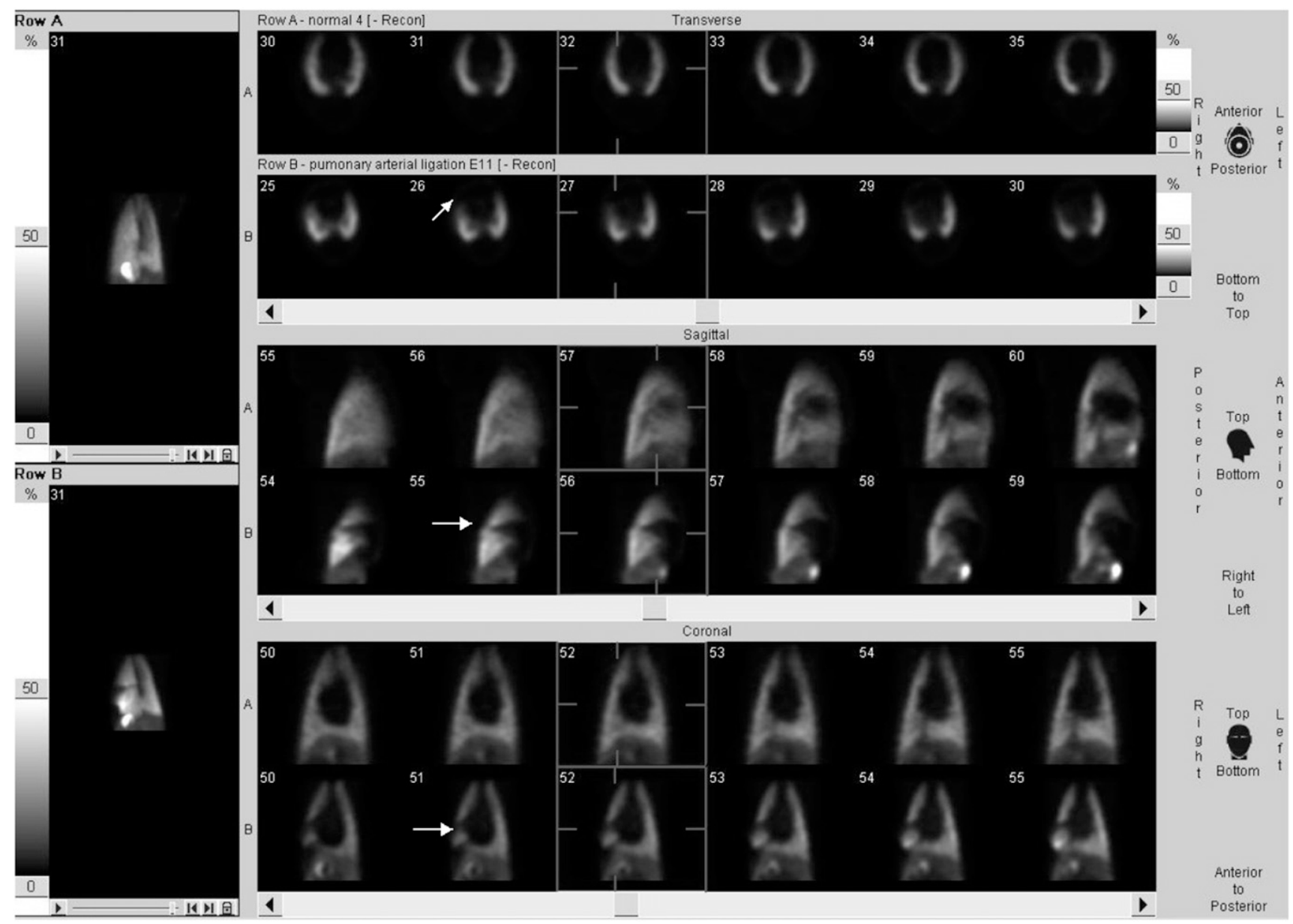

FIGURE 7. Imaging of pulmonary vascular perfusion defects with 99mTc-L-AM perfusion scan in dogs after 60 min. Tomographic images in transverse, sagittal, and coronal views are shown for a control dog and a dog that underwent right middle lobar pulmonary artery ligation. Line marker in each image indicates level of section. Images on left represent maximum-intensity projections of reconstructed tomographic images.

of $19 \mathrm{nM}(95 \% \mathrm{CI}, 9-39)$ and $70 \mathrm{nM}(95 \% \mathrm{CI}, 32-140)$, respectively.

\section{DISCUSSION}

We evaluated the potential utility of AM derivatives for molecular imaging of the pulmonary circulation. We found that intravenously injected radiolabeled AM derivatives rapidly disappeared from the circulation through predominantly pulmonary uptake, with a slow decline in lung activity over time. These properties allowed good-quality external imaging with a nuclear medicine camera and resulted in the detection of perfusion defects.

Lung perfusion scans are widely used for the diagnosis of pulmonary embolism (16). The only agent currently available for scintigraphic lung imaging is ${ }^{99 \mathrm{~m}} \mathrm{Tc}$-labeled albumin macroaggregates ( $\left.{ }^{99 \mathrm{~m}} \mathrm{Tc}-\mathrm{MAA}\right)$. These human-derived particles, varying in size from 10 to $90 \mu \mathrm{M}$, become physically trapped in small pulmonary vessels, thereby allowing external detection. The use of ${ }^{99 m} \mathrm{Tc}-\mathrm{MAA}$ is therefore limited to the detection of anatomic lung perfu- sion defects; because the agent does not have access to nonperfused regions of the lungs, there is a diagnostic "cold zone" on lung scans.

In the present study, we used ${ }^{99 \mathrm{~m}} \mathrm{Tc}$-labeled AM derivatives for molecular imaging of the pulmonary circulation. Previous studies established that the lungs are a primary site of AM clearance $(5,6)$. With ${ }^{125} \mathrm{I}-\mathrm{AM}$ as a tracer, the high binding affinity for dog lungs in vivo was evidenced by $36 \%$ first-pass clearance (7). This value is comparable to the first-pass extraction of $30 \% \pm 5 \%$ found in the present study with L-AM. We previously demonstrated that this represents specific binding as ${ }^{125} \mathrm{I}-\mathrm{AM}$ clearance by the lungs of rats is displaced by AM (7).

In the present study, we also confirmed the competitive binding of L-AM and C-AM to human cells known to express AM receptors. Unlike the binding of ${ }^{99 \mathrm{~m} T c-M A A,}$ the binding of ${ }^{99 \mathrm{~m}} \mathrm{Tc}-\mathrm{AM}$ is a biologic process dependent on the density and affinity of specific AM receptors $(8,17)$. The latter agent therefore has the potential to image not only anatomic perfusion defects but also functional defects that could modify AM receptor density or affinity. 
Using L-AM, we confirmed the potential utility of ${ }^{99 m}$ Tc-labeled AM derivatives for the diagnosis of anatomic perfusion defects mimicking pulmonary embolism. We obtained good-quality imaging with easily detectable perfusion defects. Future studies are needed to determine whether ${ }^{99 \mathrm{~m}}$ Tc-labeled AM derivatives might be valuable for the detection and quantification of conditions affecting not only the density but also the activity of AM receptors. Some preclinical as well as clinical data do suggest that the lung uptake of AM could be reduced in various conditions. For example, in a sepsis model, increased circulating AM levels are accompanied by reduced levels of expression of lung AM receptors, suggesting that the reduced clearance could contribute to the elevated circulating AM levels (18). However, it is currently not known whether AM receptor activity is specifically modified in the pulmonary circulation in various cardiovascular disorders, such as pulmonary hypertension of various etiologies and heart failure. Whether such an imaging modality might be useful for conditions other than vascular perfusion defects remains hypothetical and subject to further research.

\section{CONCLUSION}

Chelated AM derivatives displayed a short plasma halflife with prolonged predominantly lung uptake, allowing external lung perfusion imaging. These agents can be used to detect anatomic perfusion defects and have the added potential for functional vascular imaging. Compared with the natural cyclic form of the peptide, the linear form of AM allows direct labeling and displays a higher level of early lung retention as well as a higher level of urinary elimination-properties that make this derivative a more attractive imaging agent.

\section{ACKNOWLEDGMENTS}

This work was supported in part by the Program of Intellectual Property Management from the Canadian Institutes for Health Research and by PulmoScience Inc., Montreal, Quebec, Canada. François Harel is a junior scholar at the Fonds de la Recherche en Santé du Québec. Jocelyn Dupuis is a national researcher at the Fonds de la Recherche en Santé du Québec.

\section{REFERENCES}

1. Hinson JP, Kapas S, Smith DM. Adrenomedullin, a multifunctional regulatory peptide. Endocr Rev. 2000;21:138-167.

2. Sugo S, Minamino N, Kangawa K, et al. Endothelial cells actively synthesize and secrete adrenomedullin. Biochem Biophys Res Commun. 1994;201:1160-1166.

3. Hirayama N, Kitamura K, Imamura T, Kato J, Koiwaya Y, Eto T. Secretion and clearance of the mature form of adrenomedullin in humans. Life Sci. 1999;64: 2505-2509.

4. Gibbons C, Dackor R, Dunworth W, Fritz-Six K, Caron KM. Receptor activitymodifying proteins: RAMPing up adrenomedullin signaling. Mol Endocrinol. 2007;21:783-796.

5. Yanagawa B, Nagaya N. Adrenomedullin: molecular mechanisms and its role in cardiac disease. Amino Acids. 2007;32:157-164.

6. Lippton H, Chang JK, Hao Q, Summer W, Hyman AL. Adrenomedullin dilates the pulmonary vascular bed in vivo. J Appl Physiol. 1994;76:2154-2156.

7. Dupuis J, Caron A, Ruel N. Biodistribution, plasma kinetics and quantification of single-pass pulmonary clearance of adrenomedullin. Clin Sci (Lond). 2005; 109:97-102.

8. Dschietzig T, Azad HA, Asswad L, et al. The adrenomedullin receptor acts as clearance receptor in pulmonary circulation. Biochem Biophys Res Commun. 2002;294:315-318.

9. Nishikimi T, Kitamura K, Saito Y, et al. Clinical studies on the sites of production and clearance of circulating adrenomedullin in human subjects. Hypertension. 1994;24:600-604.

10. Hagner S, Haberberger R, Hay DL, et al. Immunohistochemical detection of the calcitonin receptor-like receptor protein in the microvasculature of rat endothelium. Eur J Pharmacol. 2003;481:147-151.

11. Hagner S, Stahl U, Knoblauch B, McGregor GP, Lang RE. Calcitonin receptorlike receptor: identification and distribution in human peripheral tissues. Cell Tissue Res. 2002;310:41-50.

12. Martinez A, Miller MJ, Catt KJ, Cuttitta F. Adrenomedullin receptor expression in human lung and in pulmonary tumors. J Histochem Cytochem. 1997;45:159-164.

13. Owji AA, Smith DM, Coppock HA, et al. An abundant and specific binding site for the novel vasodilator adrenomedullin in the rat. Endocrinology. 1995;136: 2127-2134.

14. Dupuis J, Goresky CA, Fournier A. Pulmonary clearance of circulating endothelin-1 in dogs in vivo: exclusive role of ETB receptors. J Appl Physiol. 1996;81:1510-1515.

15. Miller MJ, Martinez A, Unsworth EJ, et al. Adrenomedullin expression in human tumor cell lines: its potential role as an autocrine growth factor. $J$ Biol Chem. 1996;271:23345-23351.

16. Dalen JE. Pulmonary embolism: what have we learned since Virchow? Treatment and prevention. Chest. 2002;122:1801-1807.

17. Hay DL, Smith DM. Adrenomedullin receptors: molecular identity and function. Peptides. 2001;22:1753-1763.

18. Ono Y, Okano I, Kojima M, Okada K, Kangawa K. Decreased gene expression of adrenomedullin receptor in mouse lungs during sepsis. Biochem Biophys Res Commun. 2000;271:197-202. 